*akls View/Frint Document Cover Sheet tow

This document was retrieved from the Boeing ISEARCH System.

Accession \#: D196050130

Document \#: SD-FF-TA-040

Title/Desc:

RADIOLOGICAL CONSEQUENCES OF A POSTULATED DROP OF A MAXIMALLY LOADED FFTF FUEL CASK 
$\boldsymbol{H}$

Shet 22,1995

ENGINEERING DATA TRANSMITTAL

Page 1 of 1 ittation 35

i. EDT 605973

\begin{tabular}{|l|l|l|}
\hline $\begin{array}{l}\text { 2. To: (Receiving Organization) } \\
\text { FFTF Engineering/18200 }\end{array}$ & $\begin{array}{l}\text { 3. From: (originating Organization) } \\
\text { Consequence Analysis } / 8 M 400\end{array}$ & 4. Related EDT Mo.: \\
\hline $\begin{array}{l}\text { 5. Proj./Prog./Bept./Biv.: } \\
\text { FFTF }\end{array}$ & $\begin{array}{l}\text { 6. Cog. Engr.: } \\
\text { P. L. Scott }\end{array}$ & 7. Purchuse Order Mo.: \\
\hline
\end{tabular}

8. Originator Remarks:

9. Equip./Component Mo.:

This document is for Approval and Release.

10. System/Bldg./Facility: FFTF

11. Receiver Renarks:

12. Major Assm. Dug. Mo.:

13. Permit/Permit Application No.:

14. Required Response Date:

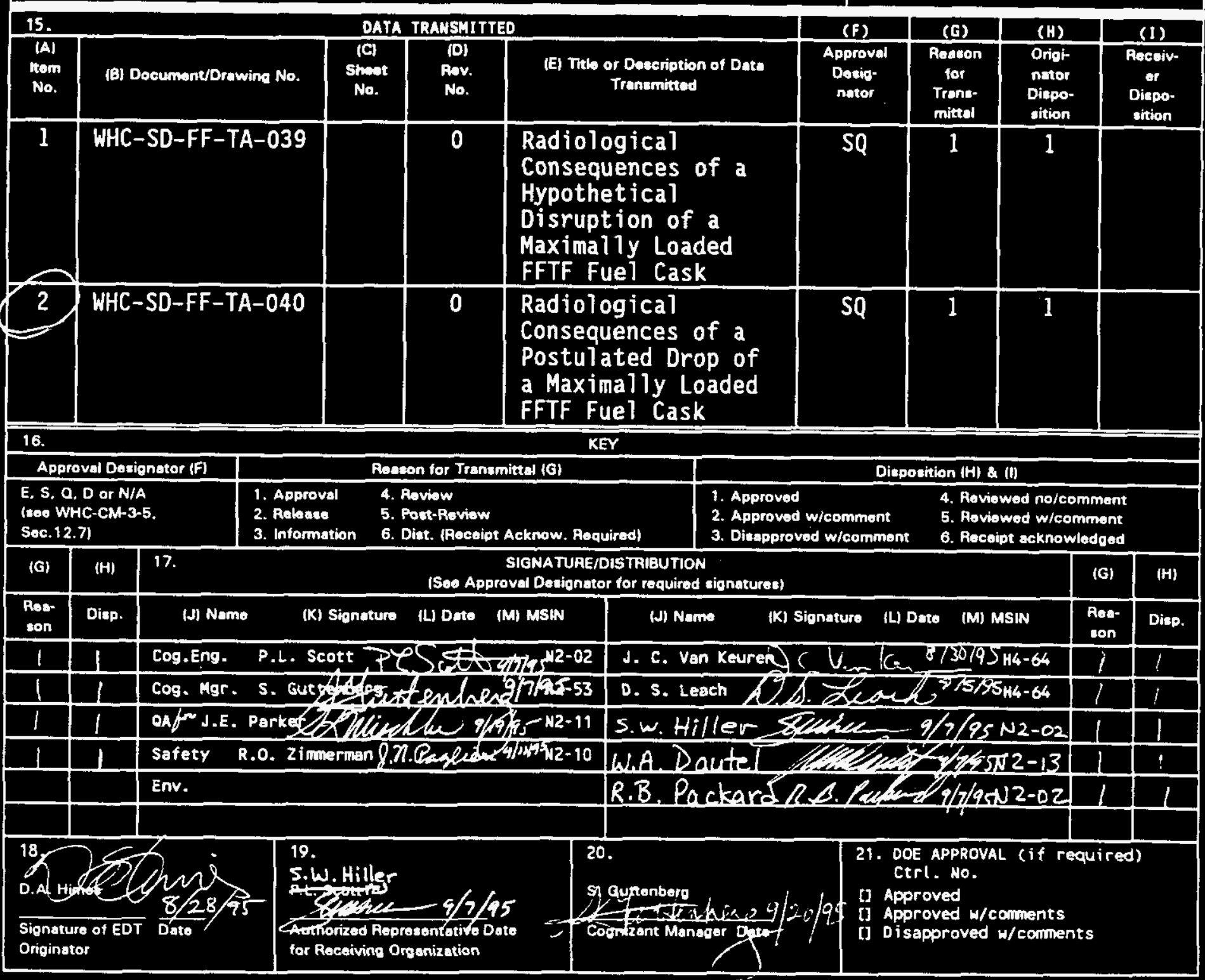

BO-7400-172-2 (04/94) GEF097 


\section{RELEASE AUTHORIZATION}

Document Number: WHC-SD-FF-TA-040, Rev. 0

Document Title: $\quad$ Radiological Consequences of a Postulated Drop of a Maximally Loaded FFTF Fuel Cask

Release Date: $\quad 9 / 22 / 95$

This document was reviewed following the procedures described in WHC-CM-3-4 and is:

APPROVED FOR PUBLIC RELEASE

WHC Information Release Administration Specialist:

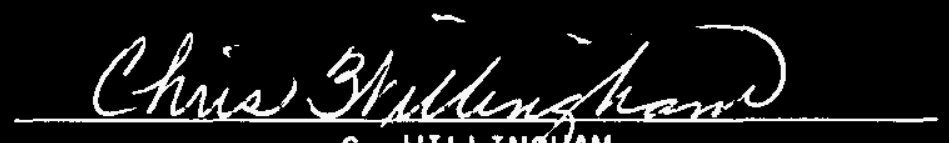

$9 / 22 / 95$

\section{c. WILLINGAM}

TRADEMARK DISCLAIMER. Reference here in to any specific commercial product, process, or service by trade name, trademark, manufacturer, or otherwise, does not necessarily constitute or imply its endorsement, recommendation, or favoring by the United States Government or any agency thereof or its contractors or subcontractors.

This report has been reproduced from the best available copy. Available in paper copy. Printed in the United States of America. To obtain copies of this report, contact:

Westinghouse Hanford Company - Document Control Services

P.0. Box 1970, Mailstop H6-08, Richland, WA 99352

Telephone: (509) 372-2420; Fax: (509) 376-4989 


\begin{tabular}{|l|l|c|}
\hline $\begin{array}{l}\text { 2. Title } \\
\text { Radiological Consequences of a Postul ated Drop of } \\
\text { a Maximally Loaded FFTF Fuel Cask }\end{array}$ & $\begin{array}{l}\text { 3. Number } \\
\text { WHC-SD-FF-TA-040 No. }\end{array}$ \\
\hline $\begin{array}{l}\text { 5. Key Hords } \\
\text { ISC, FFTF }\end{array}$ & 6. Author \\
\hline
\end{tabular}

\section{Abstract}

Onsite and site boundary radiological consequences were estimated for a postulated accidental drop of an Interim Storage Cask (ISC) loaded with 7 assemblies at the maximum available burnup. The postulated cask drop was assumed to occur from the maximum physically attainable height during crane movement of the cask. The resulting onsite and site boundary doses of $45 \mathrm{mSv}$ and $0.04 \mathrm{mSv}$ are far below the corresponding 1 Sv and 250 mSv risk guidelines for highly unlikely accidents.

8.

RELEASE STAMP

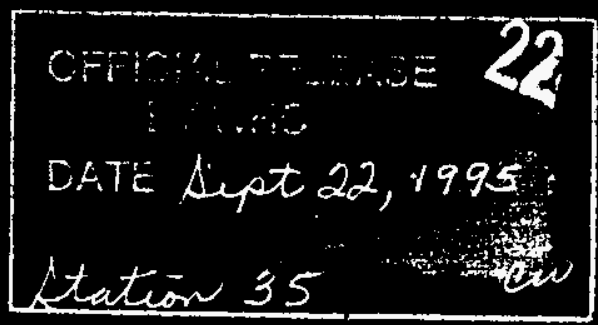


WHC-SD-FF-TA-040

Rev. 0

Page 1 of 18

\title{
RADIOLOGICAL CONSEQUENCES OF A POSTULATED DROP OF A MAXIMALLY LOADED FFTF FUEL CASK
}

\author{
D.A. Himes \\ $8 / 21 / 95$
}

\begin{abstract}
Radiological consequences onsite and at the site boundary are required for a postulated accidental drop of an Interim Storage Cask (ISC) loaded with 7 assemblies at the maximum available burnup. Several assemblies exist in the inventory with average burnups approaching 200,000 MWd/MTHM. The maximum assembly is the ACO-4 experiment with 199,400 MWd/MTHM average burnup. There are a few metal fuel assemblies and some carbide pins from $A C-3$ and $A C N-1$, however these are expected to be bounded by the high burnup driver fuel assemblies assumed for this analysis.
\end{abstract}

The postulated cask drop is to occur from the maximum physically attainable height during crane movement of the cask. The assemblies or pin containers will be contained within a Core Component Container (CCC) which will be placed inside the ISC. The ISC is fabricated of reinforced concrete with a $1.5 \mathrm{in}$. thick stainless steel liner, and is equipped with a crushable pad impact limiter which supports the CCC. In spite of the presence of the impact limiter, the postulated drop is assumed to mechanically shock the fuel enough to cause cracking in $100 \%$ of the pins and to crush and expose $1 \%$ of the fuel material. Such an impact, however, is not expected to be able to produce a major escape path out of the cask. Instead, such an impact would, at most, produce some possible cracked welds or a lid seal failure.

\section{Source Term Development:}

The cask drop is conservatively assumed to produce failure of the seven fuel assemblies similar to, but less severe than, a previously analyzed hypothetical cask disruption accident (Himes 1993). 100\% of the pins were assumed to fail in the fueled region, and, as before, $100 \%$ of the noble gases and tritium and $5 \%$ of the halogens were assumed to escape from the cask. However, only $1 \%$ of the pellets were assumed to be crushed and exposed, and only $1 \%$ of the particulates released from the fuel pins were assumed to follow the tortuous path out of the Core Component Container and the damaged Interim Storage Cask to the environment. The release fractions in terms of the inventory in one assembly were developed as follows.

Noble gases and tritium: $\quad 100 \%$ of pins breached $100 \%$ release

$\Rightarrow$ Net release fraction $=1.0 E+0$ 
WHC-SD-FF-TA-040

Rev. 0

Page 2 of 18

Halogens:

$100 \%$ of pins breached $10 \%$ release (cold fuel) $50 \%$ plateout

$\Rightarrow$ Net release fraction $=5.0 \mathrm{E}-2$

Volatile solids (Cd \& CS): $\quad 100 \%$ of pins breached

$1 \%$ of pellets crushed and exposed

$5 \%$ release from exposed pellets

$1 \%$ release through cracks in cask

$\Rightarrow$ Net release fraction $=5.0 \mathrm{E}-6$

Nonvolatile solids:

$100 \%$ of pins breached

$1 \%$ of pellets crushed and exposed

$1 \%$ release from exposed pellets

$5 \%$ respirable $(\leq 10 \mu \mathrm{m})$

$1 \%$ release through cracks in cask

$\Rightarrow$ Net release fraction $=5.0 \mathrm{E}-8$

The $1 \%$ of pellets crushed and exposed must have the cladding actually removed or cracked and grossly deformed, since a simple crack in the cladding, while allowing the passage of gas, is not usually wide enough to allow passage of a significant amount of particulate material. The $5 \%$ respirable fraction corresponds to the particle fraction small enough to reach the lower lungs. It is permissible to take credit for this fraction in this case since nearly all of the dose from the nonvolatile particles is due to the transuranics (for which the nonrespirable fraction does not produce a significant dose).

The inventory per assembly was derived from an ORIGEN2 run for a standard driver with an average burn-up of 150,000 MWd/MTHM which has decayed for 5 years to a decay heat rate of approximately $250 \mathrm{~W}$ (Nguyen 1992, Simons 1991). The resulting releases are shown in Table 1 .

Table 1: Releases to environment from one fuel assembly due to postulated cask drop

\begin{tabular}{lcc} 
Isotope & $\begin{array}{c}\text { 1 Assembly } \\
\text { Inventory (Ci) }\end{array}$ & $\begin{array}{c}\text { Release due to } \\
\text { 1 Assembly (Ci) }\end{array}$ \\
\hline H 3 & $7.82 \mathrm{E}+1$ & $7.8 \mathrm{E}+1$ \\
KR85 & $6.74 \mathrm{E}+2$ & $6.74+2$ \\
SR90 & $5.04 \mathrm{E}+3$ & $2.5 \mathrm{E}-4$ \\
Y 90 & $5.05 \mathrm{E}+3$ & $2.5 \mathrm{E}-4$ \\
TC99 & $1.99 \mathrm{E}+0$ & $1.0 \mathrm{E}-7$ \\
RU106 & $4.89 \mathrm{E}+3$ & $2.5 \mathrm{E}-4$ \\
AG110M & $8.89 \mathrm{E}+0$ & $4.5 \mathrm{E}-7$ \\
CD113M & $1.29 \mathrm{E}+1$ & $6.5 \mathrm{E}-5$
\end{tabular}


WHC-SD-FF-TA-040

Rev. 0

Page 3 of 18

\begin{tabular}{lll} 
Isotope & $\begin{array}{c}\text { 1 Assembly } \\
\text { Inventory (Ci) }\end{array}$ & $\begin{array}{c}\text { Release due to } \\
\text { 1 Assembly (Ci) }\end{array}$ \\
\hline \hline SB125 & $1.21 \mathrm{E}+3$ & $6.1 \mathrm{E}-5$ \\
TE125M & $2.95 \mathrm{E}+2$ & $1.5 \mathrm{E}-5$ \\
I 129 & $6.63 \mathrm{E}-3$ & $3.3 \mathrm{E}-4$ \\
CS134 & $2.51 \mathrm{E}+3$ & $1.3 \mathrm{E}-2$ \\
CS137 & $1.37 \mathrm{E}+4$ & $6.9 \mathrm{E}-2$ \\
CE144 & $1.66 \mathrm{E}+3$ & $8.3 \mathrm{E}-5$ \\
PR144M & $1.99 \mathrm{E}+1$ & $1.0 \mathrm{E}-6$ \\
PR144 & $1.66 \mathrm{E}+3$ & $8.3 \mathrm{E}-5$ \\
PM147 & $8.73 \mathrm{E}+3$ & $4.4 \mathrm{E}-4$ \\
SM151 & $5.21 \mathrm{E}+2$ & $2.6 \mathrm{E}-5$ \\
EU154 & $5.08 \mathrm{E}+2$ & $2.5 \mathrm{E}-5$ \\
EU155 & $1.15 \mathrm{E}+3$ & $5.8 \mathrm{E}-5$ \\
PU238 & $2.81 \mathrm{E}+2$ & $1.4 \mathrm{E}-5$ \\
PU239 & $3.49 \mathrm{E}+2$ & $1.8 \mathrm{E}-5$ \\
PU240 & $2.68 \mathrm{E}+2$ & $1.3 \mathrm{E}-5$ \\
PU241 & $1.46 \mathrm{E}+4$ & $7.3 \mathrm{E}-4$ \\
AM241 & $2.53 \mathrm{E}+2$ & $1.3 \mathrm{E}-5$ \\
AM242M & $1.93 \mathrm{E}+1$ & $9.7 \mathrm{E}-7$ \\
AM242 & $1.93 \mathrm{E}+1$ & $9.7 \mathrm{E}-7$ \\
CM242 & $2.04 \mathrm{E}+1$ & $1.0 \mathrm{E}-6$ \\
CM244 & $2.04 \mathrm{E}+1$ & $1.0 \mathrm{E}-6$
\end{tabular}

\section{Receptor Descriptions:}

Onsite:

For a ground level release, this receptor is normally at a distance of $100 \mathrm{~m}$ in the worst direction. Doses calculated for this receptor include inhalation and submersion. This release is assumed to be of short duration so no evacuation is assumed.

Site Boundary:

This receptor is located at the site boundary in the worst direction and is assumed to stay at this location for the duration of the accident. Doses calculated include inhalation and submersion. In sectors where the site is bounded by the Columbia River, this receptor is assumed to be located on the near bank of the river. For this analys is, the receptor is evaluated for both the present and proposed site boundaries.

Agricultural Area:

This is the residence of the ingestion pathway receptor (IPR). This receptor is assumed to grow his own food, including a variety of crops, meat and dairy products and to continue to do so at this location for 50 years following the accident. No credit is taken for uncontaminated foodstuffs brought in from outside the area. Note that potential 
WHC-SD-FF-TA-040

Rev. 0

Page 4 of 18

ingestion and ground shine doses are not included in the comparison to risk guidelines (WHC, 1988) since, in the case of an accident, any contaminated land or products would not be used, and ingestion and ground shine would not, therefore, be actual exposure pathways. The release is assumed to occur just prior to the autumn harvest in order to maximize consequences with regard to the time of the accident. In sectors where the site is bounded by the Columbia River, this receptor is assumed to be located on the far bank of the river. For this analysis, the IPR is evaluated for both the present and proposed site boundaries.

\section{TRANSPORT ASSUMPTIONS:}

Acute 99.5 percentile ground level release dispersion factors $(X / Q)$ were generated using the GXQ code (Hey 1994) at the 16 sectors at $100 \mathrm{~m}$ and at the 16 sector locations around both the present and proposed site boundaries. These results are summarized in tables 2 and 3 below. The asterisks identify the worst case locations.

Table 2: Onsite $99.5 \% \times / Q$ s at $100 \mathrm{~m}$ for releases in the 400 Area

\begin{tabular}{cc} 
Sector & $X / Q\left(\mathrm{~s} / \mathrm{m}^{3}\right)$ \\
\hline S & $\star 3.17 \mathrm{E}-2 *$ \\
SSW & $2.32 \mathrm{E}-2$ \\
SW & $1.46 \mathrm{E}-2$ \\
WSW & $1.05 \mathrm{E}-2$ \\
W & $1.43 \mathrm{E}-2$ \\
WWW & $1.25 \mathrm{E}-2$ \\
NW & $1.53 \mathrm{E}-2$ \\
NNW & $2.37 \mathrm{E}-2$ \\
N & $* 3.17 \mathrm{E}-2 *$ \\
NNE & $2.62 \mathrm{E}-2$ \\
NE & $1.68 \mathrm{E}-2$ \\
ENE & $1.50 \mathrm{E}-2$ \\
E & $1.73 \mathrm{E}-2$ \\
ESE & $1.61 \mathrm{E}-2$ \\
SE & $2.66 \mathrm{E}-2$ \\
SSE & $2.56 \mathrm{E}-2$ \\
a11 (95\%) & $3.11 \mathrm{E}-2$
\end{tabular}


WHC-SD-FF-TA-040

Rev. 0

Page 5 of 18

Table 3: Acute $99.5 \% \times / Q$ values at site boundary locations for ground level release at FFTF complex

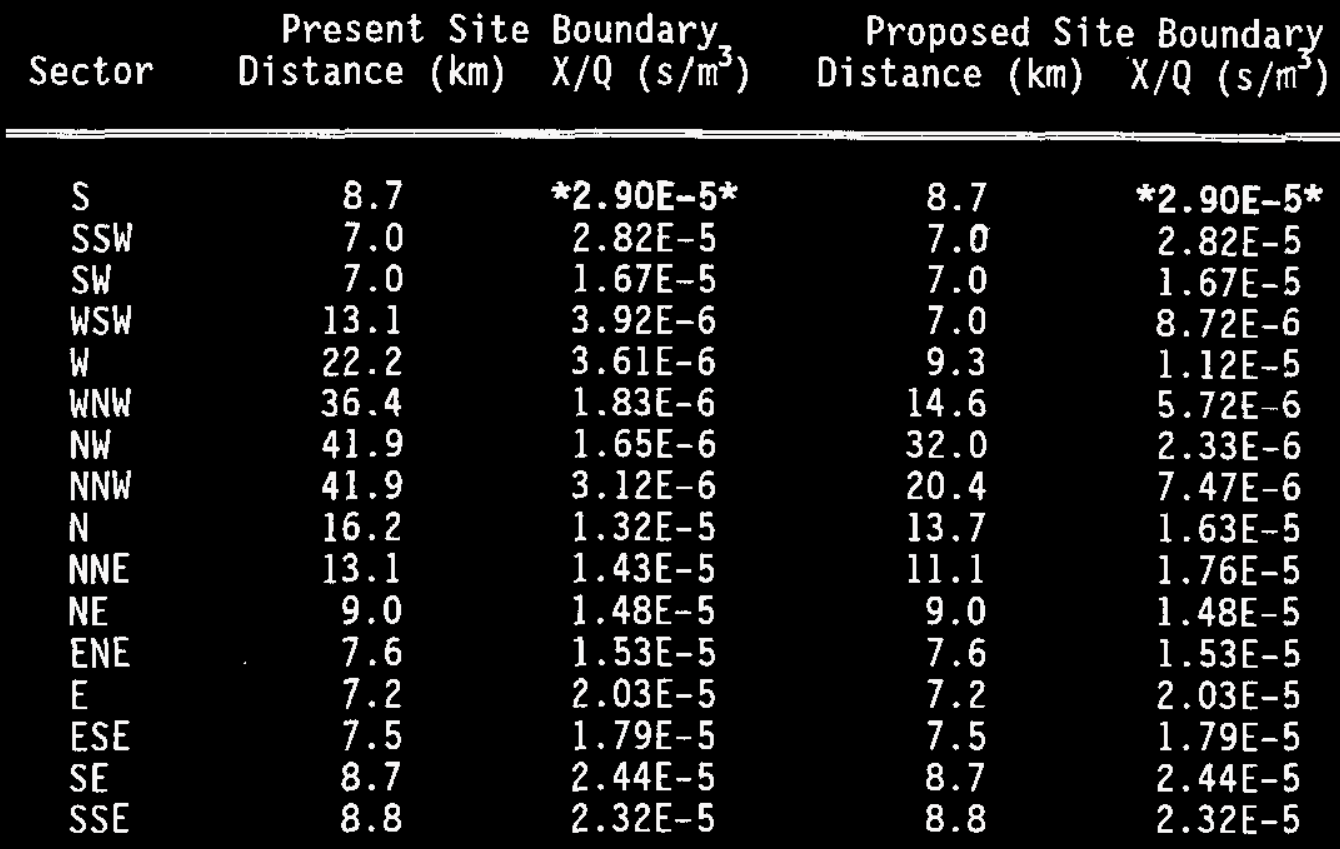

Code Documentation:

GXQ version $4.0(12 / 19 / 94)$ (Hey 1994)

Joint Frequency Data: 200 Areas, $10 \mathrm{~m}$, Pasquill A-G (1983-1991 Average)

GXQ input/output files are attached for reference.

GENII version $1.485(12 / 3 / 90)$ (Napier 1988)

GENI I Default Parameter Values $(3 / 28 / 90)$

Radionuclide Library - Times $<100$ years $(7 / 23 / 93)$

External Dose Factor Library (5/8/90)

Worst-Case Solubilities, Yearly Dose Increments (7/23/93)

Typical GENII input file is attached for reference.

\section{RESULTS:}

The resulting effective dose equivalents (EDE) for releases due to 1 fuel assembly at an average burnup of 150,000 MWd/MTHM decayed to $250 \mathrm{~W}$ (inventory shown in Table 1) are shown in Table 4. 
WHC-SD-FF-TA-040

Rev. 0

Page 6 of 18

Table 4: Resulting doses for postulated cask drop due to

1 assembly with inventory shown in Table 1

Receptor Dose Type Effective Dose Equivalent EDE

\begin{tabular}{|c|c|c|c|}
\hline Receptor & Dose Type & (rem) & (mSv \\
\hline $\begin{array}{c}\text { Onsite } \\
(100 \mathrm{~m} \mathrm{~S} \text { or } \mathrm{N})\end{array}$ & $\begin{array}{l}\text { Inhalation } \\
\text { Submersion } \\
\text { Total }\end{array}$ & $\begin{array}{r}4.4 \mathrm{E}-1 \\
1.2 \mathrm{E}-2 \\
4.5 \mathrm{E}-1\end{array}$ & $\begin{array}{l}4.4 \mathrm{E}+0 \\
1.2 \mathrm{E}-1 \\
4.5 \mathrm{E}+0\end{array}$ \\
\hline $\begin{array}{l}\text { Site Boundary } \\
(8.7 \mathrm{~km} \mathrm{~S})\end{array}$ & $\begin{array}{l}\text { Inhalation } \\
\text { Submersion } \\
\text { Total }\end{array}$ & $\begin{array}{l}3.9 \mathrm{E}-4 \\
1.1 \mathrm{E}-5 \\
4.0 \mathrm{E}-4\end{array}$ & $\begin{array}{l}3.9 \mathrm{E}-3 \\
1.1 \mathrm{E}-4 \\
4.0 \mathrm{E}-3\end{array}$ \\
\hline $\begin{array}{c}\text { IPR } \\
(8.7 \mathrm{~km} \mathrm{~S})\end{array}$ & $\begin{array}{l}\text { Inhalation } \\
\text { Submersion } \\
\text { Ingestion } \\
\text { Ground Shine } \\
\text { Total }\end{array}$ & $\begin{array}{l}3.9 \mathrm{E}-4 \\
1.1 \mathrm{E}-5 \\
2.2 \mathrm{E}-2 \\
4.2 \mathrm{E}-4 \\
2.3 \mathrm{E}-2\end{array}$ & $\begin{array}{l}3.9 \mathrm{E}-3 \\
1.1 \mathrm{E}-4 \\
2.2 \mathrm{E}-1 \\
4.2 \mathrm{E}-3 \\
2.3 \mathrm{E}-1\end{array}$ \\
\hline
\end{tabular}

About $74 \%$ of the total dose shown above is due to the TRU isotopes. Of the remaining $26 \% \mathrm{Cs}-134$ and $\mathrm{Cs}-137$ account for about $6 \%$ with the remainder being almost entirely due to the tritium (17\%) and $\mathrm{kr}-85(3 \%)$. Once the decay heat of an assembly has decreased enough (due to decay of short-live fission products) to be handled and stored dry, most of the dose is due to the longlived TRU and Cesium isotopes, and tends to increase slowly. with decay time due to buildup of Am-241. The 5 year decayed fuel represented by the inventory in Table 1 will therefore bound the same fuel decayed 4 years.

The doses shown above in Table 3 must be multiplied by 7 to account for the 7 assemblies in the maximally loaded cask. In addition, an adjustment must be made for the higher burnup of some of the assemblies which could be in the cask. The highest average burnup of the available assemblies is 199,400 MWd/MTHM for ACO-4. This is a factor of 1.33 higher than the burnup assumed for Table 3. The doses per assembly were therefore adjusted for burnup by simply multiplying by 1.33 . This is extremely conservative since the dose does not go to zero for zero burnup, and there are relatively few assemblies with burnups above 150,000 MWd/MTHM. Along with the multiplier of 7 for the number of assemblies in the cask, the total adjustment factor for the doses shown in Table 3 is, therefore, 9.3. For simplicity and added conservatism, the doses in Table 3 were multiplied by a factor of 10 . This should also be enough adjustment to account for the minor variations in enrichment, loading, etc. in the test assemblies. The results are shown below in Table 5 for the maximally loaded cask. 
WHC-SD-FF-TA-040

Rev. 0

Page 7 of 18

Table 5: Resulting doses for postulated drop of a maximally loaded cask

\begin{tabular}{|c|c|c|c|}
\hline Receptor & Dose Type & $\begin{array}{l}\text { Effective Dose } \\
\text { (rem) }\end{array}$ & $\begin{array}{c}\text { Equivalent } \\
(m S \mathrm{EDE})\end{array}$ \\
\hline $\begin{array}{c}\text { Onsite } \\
(100 \mathrm{~m} \mathrm{~S} \text { or } \mathrm{N})\end{array}$ & $\begin{array}{l}\text { Inhalation } \\
\text { Submersion } \\
\text { Total }\end{array}$ & $\begin{array}{l}4.4 \mathrm{E}+0 \\
1.2 \mathrm{E}-1 \\
4.5 \mathrm{E}+0\end{array}$ & $\begin{array}{l}4.4 \mathrm{E}+1 \\
1.2 \mathrm{E}+0 \\
4.5 \mathrm{E}+1\end{array}$ \\
\hline $\begin{array}{l}\text { Site Boundary } \\
(8.7 \mathrm{~km} \mathrm{~S})\end{array}$ & $\begin{array}{l}\text { Inhalation } \\
\text { Submersion } \\
\text { Total }\end{array}$ & $\begin{array}{r}3.9 \mathrm{E}-3 \\
1.1 \mathrm{E}-4 \\
4.0 \mathrm{E}-3\end{array}$ & $\begin{array}{l}3.9 \mathrm{E}-2 \\
1.1 \mathrm{E}-3 \\
4.0 \mathrm{E}-2\end{array}$ \\
\hline $\begin{array}{c}\text { IPR } \\
(8.7 \mathrm{~km} \mathrm{~S})\end{array}$ & $\begin{array}{l}\text { Inhalation } \\
\text { Submersion } \\
\text { Ingestion } \\
\text { Ground Shine } \\
\text { Total }\end{array}$ & $\begin{array}{l}3.9 \mathrm{E}-3 \\
1.1 \mathrm{E}-4 \\
2.2 \mathrm{E}-1 \\
4.2 \mathrm{E}-3 \\
2.3 \mathrm{E}-1\end{array}$ & $\begin{array}{l}3.9 \mathrm{E}-2 \\
1.1 \mathrm{E}-3 \\
2.2 \mathrm{E}+0 \\
4.2 \mathrm{E}-2 \\
2.3 \mathrm{E}+0\end{array}$ \\
\hline
\end{tabular}

Both the onsite and offsite doses are far below the 1 Sv (100 rem) onsite and the $250 \mathrm{mSv}$ (25 rem) offsite radiological risk guidelines for credible events with frequencies between $10^{-4} / y$ and $10^{-6} / y$ (WHC 1988).

\section{References:}

Hey, B. E., 1994, GXQ Program User's Guide, WHC-SD-GN-SWD-30002, Rev. 1, Westinghouse Hanford Company, Rich1and, Washington, Dec. 19, 1994.

Himes, D. A., 1993, Radiological Consequences of a Hypothetical Disruption of an Interim Storage Cask (ISC), Radiological \& Toxicological Analysis, 29250-DAH-93001, January 13, 1993.

Napier, B. A., et al., 1988, GENII - The Hanford Environmental Radiation Dosimetry Software System, PNL-6584, Pacific Northwest Laboratory, Richland, Washington, November, 1988.

Nguyen, L. V., 1992, Radiological Analysis of 150,000 MNd/MTHM Spent Fuel With 250 Watt Decay Heat For A Seismically Initiated Beyond Design Basis Accident, WHC-SD-FF-TA-027 Rev. 0, May 11, 1992.

Simons, R. L., 1991, Memo to L. R. Bese1, Radiation Source Terms for the FFTF Off-Load Program, August 16, 1991.

WHC, 1988, Safety Analysis Manual, WHC-CM-4-46, Westinghouse Hanford Company, Richland Washington, September 30, 1988. 
Rev. 0

Page 8 of 18

\title{
EXQ File
}

Site Boundary

GXQ Version 4.0A

December 19, 1994

\author{
General Purpose Atmospheric Dispersion Code \\ Produced by Westinghouse Hanford Company \\ Users Guide documented in WHC-SD-GN-SWD-30002 Rev. 1. \\ Validation documented in WHC-SD-GN-SWD-30003 Rev. 1. \\ Code Custodian is: Brit E. Hey \\ Westinghouse Hanford Company \\ P.0. Box 1970 \\ Richland, WA 99352 \\ (509) 376-2921
}

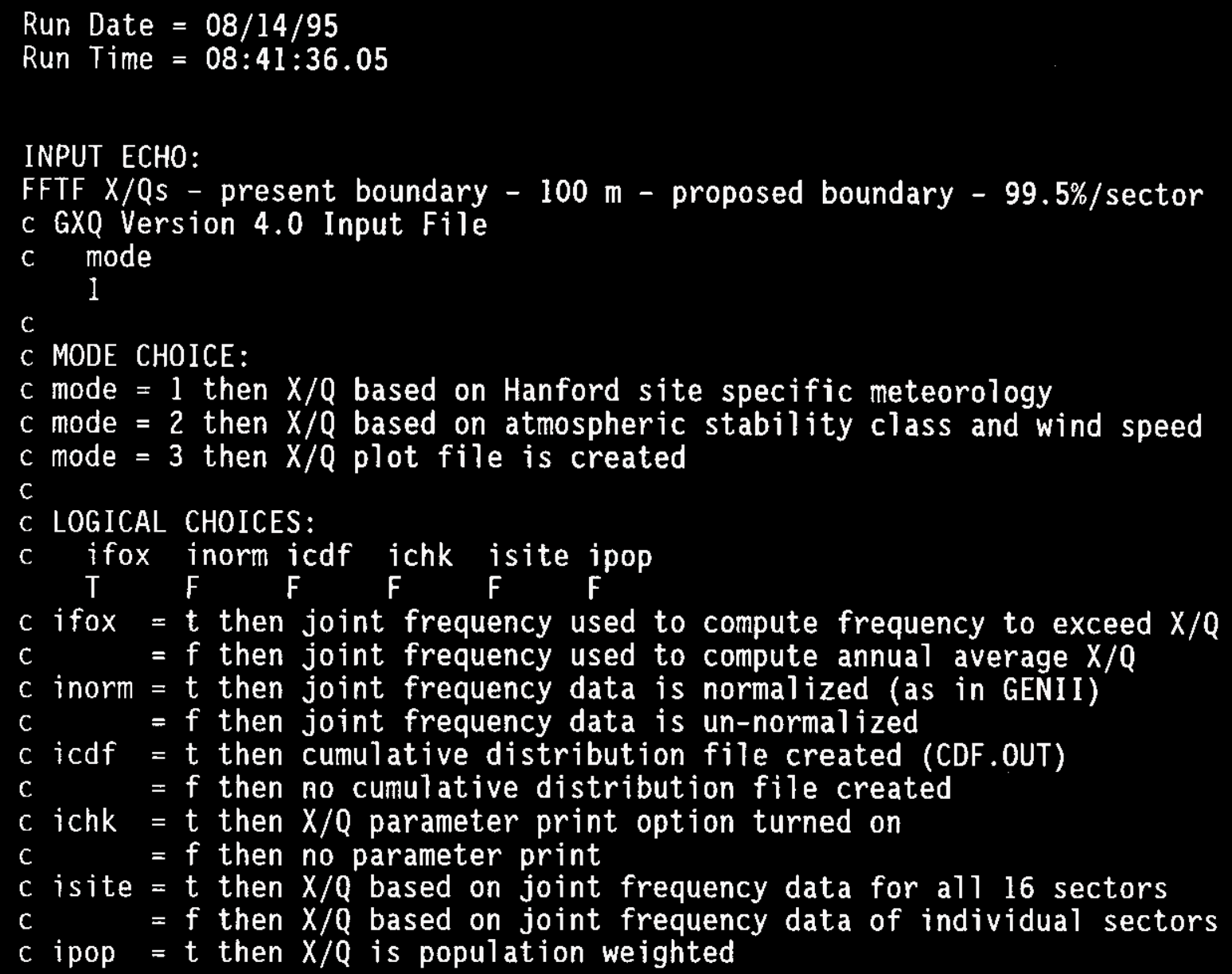


c EFFECTIVE RELEASE HEIGHT ADJUSTMENT MODELS:

C (irise igrnd) iwash igrav

$\begin{array}{llll}0 & 0 & 0 & 0\end{array}$

$c$ ipuff $=1$ then $X / Q$ calculated using puff model

$\mathrm{c} \quad=0$ then $X / Q$ calculated using default continuous $p l$ ume model

$c$ idep $=1$ then plume depletion model turned on (Chamberlain model)

$c$ isrc $=1$ then $X / Q$ multiplied by scalar

$c \quad=2$ then $X / Q$ adjusted by wind speed function

$c$ iwind $=1$ then wind speed corrected for plume height

$c$ iwake $=1$ then NRC RG 1.145 building wake model turned on

c $\quad=2$ then MACCS virtual distance building wake model turned on

$c \mathrm{ipm}=1$ then NRC RG 1.145 plume meander mode] turned on

c $\quad=2$ then 5th Power Law plume meander model turned on

c $\quad=3$ then sector average model turned on

c iflow $=1$ then sigmas adjusted for volume flow rate

$c$ ientr $=1$ then method of Pasquill used to account for entrainment

$c$ irise $=1$ then MACCS buoyant plume rise model turned on

c $\quad=\cdot 2$ then ISC2 momentum/buoyancy plume rise model turned on

c igrnd $=1$ then Mills buoyant plume rise modification for ground effects

c iwash $=1$ then stack downwash model turned on

c igrav = 1 then gravitational settling model turned on

$c \quad=0$ unless specified otherwise, 0 turns model off

c

c PARAMETER INPUT:

C

release

reference

height

hs (m)

anemometer

height

ha(m)

xing

height

frequency

$\overline{0.00000 E+00}$

$\overline{1.00000 E+01}$

$\mathrm{hm}(\mathrm{m})$

exceed

initial

initial

plume

width

$W b(m)$

plume

height

$\mathrm{Hb}(\mathrm{m})$

$\overline{1.00000 E+03}$

$\mathrm{Cx}(\%)$

$\overline{0.00000 E+00}$

$1.00000 \mathrm{E}+01$

release

duration

trd(hr)

$5.00000 \mathrm{E}-01$

C

initial

ambient

temperature

$\operatorname{Tamb}(C)$

plume

temperature

TO(C)

$0.00000 E+00$

initial

plume

flow rate

$\mathrm{V} 0(\mathrm{~m} 3 / \mathrm{s})$

\begin{tabular}{|c|c|}
\hline $\begin{array}{l}\text { deposition } \\
\operatorname{velocity} \\
\operatorname{vd}(\mathrm{m} / \mathrm{s})\end{array}$ & $\begin{array}{l}\text { gravitationa } \\
\text { settling } \\
\text { velocity } \\
\text { vg }(\mathrm{m} / \mathrm{s})\end{array}$ \\
\hline $1.00000 \mathrm{E}-02$ & $\overline{1.00000 \mathrm{E}-02}$ \\
\hline $\begin{array}{l}\text { release } \\
\text { diameter } \\
d(m)\end{array}$ & $\begin{array}{l}\text { convective } \\
\text { heat release } \\
\text { rate(1) } \\
\text { qh(w) }\end{array}$ \\
\hline
\end{tabular}


WHC-SD-FF-TA-040

Rev. 0

Page 10 of 18

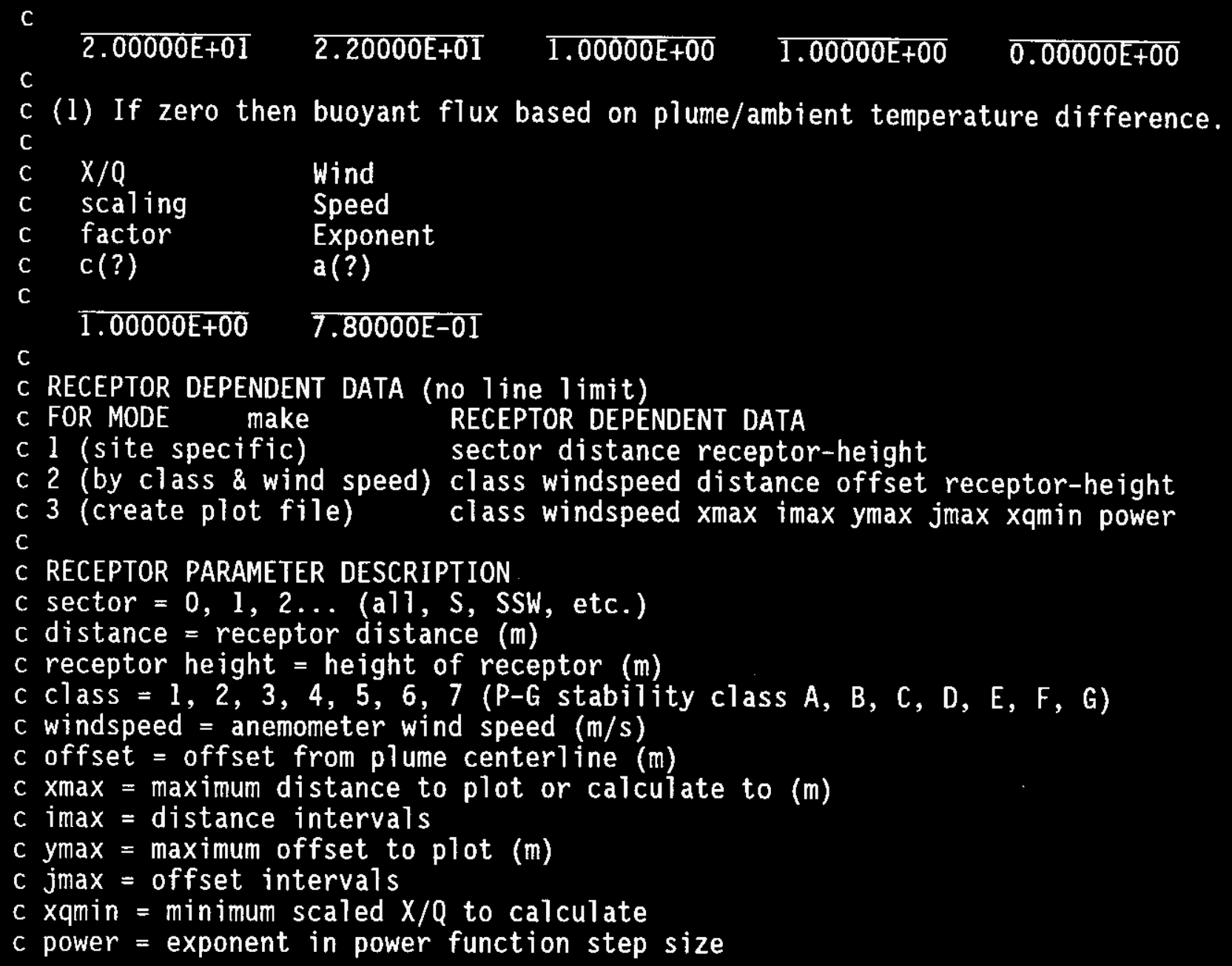


WHC-SD-FF-TA-040

Rev. 0

Page 11 of 18

FFTF $X / Q$ S - present boundary - $100 \mathrm{~m}$ - proposed boundary - $99.5 \% /$ sector

\begin{tabular}{|c|c|c|c|c|c|c|c|c|}
\hline SECTOR & $\begin{array}{l}\text { DISTANCE } \\
(\mathrm{m})\end{array}$ & $\begin{array}{c}\text { RECEPT } \\
\text { HEIGHT } \\
(\mathrm{m})\end{array}$ & $\begin{array}{c}\text { SECT. } \\
\text { FREQ. } \\
(\%)\end{array}$ & POPULATION & $\begin{array}{c}\text { TOTAL } \\
\text { POPULATION } \\
\text { SCALED } \\
X / Q \\
(\mathrm{~s} / \mathrm{m} 3)\end{array}$ & $\begin{array}{c}\text { AVERAGE } \\
\text { INDIVIDUAL } \\
\text { SCALED } \\
X / Q \\
(\mathrm{~s} / \mathrm{m} 3)\end{array}$ & $\begin{array}{l}\text { ATM. } \\
\text { STAB. } \\
\text { CLASS }\end{array}$ & $\begin{array}{l}\text { WIND } \\
\text { SPEED } \\
(\mathrm{m} / \mathrm{s})\end{array}$ \\
\hline$\overline{\mathrm{s}}$ & 8700 & 0 & 7.29 & $\bar{I}$ & $2.90 \mathrm{E}-05$ & $2.90 \mathrm{E}-05$ & $F$ & $0 . \overline{89}$ \\
\hline SSW & 7000 & 0 & 5.75 & 1 & $2.82 \mathrm{E}-05$ & $2.82 \mathrm{E}-05$ & G & 2.65 \\
\hline SW & 7000 & 0 & 3.41 & 1 & $1.67 \mathrm{E}-05$ & $1.67 \mathrm{E}-05$ & $E$ & 0.89 \\
\hline WSW & 13100 & 0 & 2.22 & 1 & $3.92 \mathrm{E}-06$ & $3.92 \mathrm{E}-06$ & $\mathrm{~F}$ & 4.70 \\
\hline & 22200 & 0 & 2.87 & 1 & $3.61 \mathrm{E}-06$ & $3.61 \mathrm{E}-06$ & $\mathrm{E}$ & 0.89 \\
\hline WNW & 36400 & 0 & 2.65 & 1 & $1.83 \mathrm{E}-06$ & $1.83 \mathrm{E}-06$ & $F$ & 2.65 \\
\hline NW & 41900 & 0 & 4.08 & 1 & $1.65 \mathrm{E}-06$ & $1.65 \mathrm{E}-06$ & E & 0.89 \\
\hline NNW & 41900 & 0 & 7.41 & I & $3.12 \mathrm{E}-06$ & $3.12 \mathrm{E}-06$ & G & 2.65 \\
\hline & 16200 & 0 & 13.26 & 1 & $1.32 E-05$ & $1.32 \mathrm{E}-05$ & $\mathrm{~F}$ & 0.89 \\
\hline NNE & 13100 & 0 & 12.15 & 1 & $1.43 E-05$ & $1.43 \mathrm{E}-05$ & G & 2.65 \\
\hline $\mathrm{NE}$ & 9000 & 0 & 6.95 & 1 & $1.48 E-05$ & $1.48 \mathrm{E}-05$ & $G$ & 4.70 \\
\hline ENE & 7600 & 0 & 3.94 & 1 & $1.53 E-05$ & $1.53 \mathrm{E}-05$ & E & 0.89 \\
\hline E & 720 & 0 & 5.45 & 1 & $2.03 \mathrm{E}-05$ & $2.03 \mathrm{E}-05$ & G & 4.70 \\
\hline ESE & 7500 & 0 & 6.48 & 1 & $1.79 E-05$ & $1.79 E-05$ & G & 4.70 \\
\hline SE & 8700 & 0 & 9.75 & 1 & $2.44 \mathrm{E}-05$ & $2.44 \mathrm{E}-05$ & G & 2.65 \\
\hline SSE & 880 & 0 & 6.32 & 1 & $2.32 \mathrm{E}-05$ & $2.32 \mathrm{E}-05$ & G & 2.65 \\
\hline 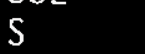 & 100 & 0 & 7.29 & i & 3.17E-02 & 3.17E-02 & 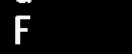 & 0.89 \\
\hline SSW & 10 & 0 & 5.75 & 1 & $2.32 \mathrm{E}-02$ & 2.32E-02 & G & 2.65 \\
\hline SW & 100 & 0 & 3.41 & i & $1.46 \mathrm{E}-02$ & $1.46 \mathrm{E}-02$ & $\bar{E}$ & 0.89 \\
\hline WSW & 100 & 0 & 2.22 & 1 & $1.05 \mathrm{E}-02$ & $1.05 \mathrm{E}-02$ & $\mathrm{~F}$ & 2.65 \\
\hline 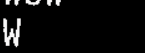 & 10 & 0 & 2.87 & i & $1.43 \mathrm{E}-02$ & $1.43 \mathrm{E}-02$ & $E$ & 0.89 \\
\hline WNW & 10 & 0 & 2.65 & 1 & $1.25 \mathrm{E}-02$ & $1.25 \mathrm{E}-02$ & $\bar{F}$ & 2.65 \\
\hline NW & 10 & 0 & 4.08 & 1 & $1.53 \mathrm{E}-02$ & $1.53 \mathrm{E}-02$ & E & 0.89 \\
\hline NNW & 100 & 0 & 7.41 & 1 & $2.37 E-02$ & $2.37 \mathrm{E}-02$ & G & 2.65 \\
\hline$N$ & 100 & 0 & 13.26 & 1 & $3.17 \mathrm{E}-02$ & $3.17 \mathrm{E}-02$ & $F$ & 0.89 \\
\hline NNE & 100 & 0 & 12.15 & I & $2.62 \mathrm{E}-02$ & $2.62 E-02$ & G & 2.65 \\
\hline NE & 100 & 0 & 6.95 & 1 & $1.68 \mathrm{E}-02$ & $1.68 \mathrm{E}-02$ & & 4.70 \\
\hline ENE & 10 & 0 & 3.94 & 1 & $1.50 \mathrm{E}-02$ & 1.50 & $E$ & 0.89 \\
\hline E & 100 & 0 & 5.45 & 1 & $1.73 \mathrm{E}-02$ & $1.73 \mathrm{E}-02$ & G & 4.70 \\
\hline ESE & 100 & 0 & 6.48 & 1 & $1.61 \mathrm{E}-02$ & $1.61 \mathrm{E}-02$ & $G$ & 4.70 \\
\hline SE & 10 & 0 & 9.75 & 1 & $2.66 \mathrm{E}-02$ & $2.66 \mathrm{E}-02$ & $G$ & 2.65 \\
\hline SSE & 10 & 0 & 6.32 & 1 & $2.56 E-02$ & $2.56 \mathrm{E}-02$ & $\bar{G}$ & 2.65 \\
\hline $\mathrm{s}$ & 870 & 0 & 7.29 & i & $2.90 \mathrm{E}-05$ & $2.90 \mathrm{E}-05$ & $\mathrm{~F}$ & 0.89 \\
\hline SSW & 7000 & 0 & 5.75 & i & $2.82 \mathrm{E}-05$ & $2.82 \mathrm{E}-05$ & $G$ & 2.65 \\
\hline SW & 700 & 0 & 3.41 & i & $1.67 \mathrm{E}-05$ & $1.67 E-05$ & $E$ & \\
\hline WSW & 700 & 0 & 2. & 1 & $8.72 \mathrm{E}-06$ & $8.72 \mathrm{E}-06$ & $\mathrm{~F}$ & 4.70 \\
\hline W & 930 & 0 & 2.87 & 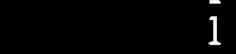 & $1.12 \mathrm{E}-05$ & $1.12 E-05$ & $E$ & 0.89 \\
\hline WNW & 146 & 0 & 2.65 & 1 & $5.72 \mathrm{E}-06$ & $5.72 \mathrm{E}-06$ & $\vec{F}$ & \\
\hline NW & 320 & 0 & 4.08 & 1 & 2.33 & 2.33 & $E$ & 0.89 \\
\hline NNW & 204 & 0 & $7.2 .5 y+3$ & 1 & $7.47 \mathrm{E}-06$ & $7.47 E-06$ & G & 2.65 \\
\hline & 13700 & 0 & 13.26 & 1 & $1.63 \mathrm{E}-05$ & $1.63 \mathrm{E}-05$ & $\mathrm{~F}$ & 0.89 \\
\hline
\end{tabular}


WHC-SD-FF-TA-040

Rev. 0

Page 12 of 18

\begin{tabular}{|c|c|c|c|c|c|c|c|}
\hline $\begin{array}{l}\text { NNE } \\
\text { NE } \\
\text { ENE } \\
\text { E } \\
\text { ESE } \\
\text { SE } \\
\text { SSE }\end{array}$ & $\begin{array}{r}11100 \\
9000 \\
7600 \\
7200 \\
7500 \\
8700 \\
8800\end{array}$ & $\begin{array}{l}0 \\
0 \\
0 \\
0 \\
0 \\
0 \\
0\end{array}$ & $\begin{array}{r}12.15 \\
6.95 \\
3.94 \\
5.45 \\
6.48 \\
9.75 \\
6.32\end{array}$ & $\begin{array}{l}1 \\
1 \\
1 \\
1 \\
1 \\
1 \\
1\end{array}$ & $\begin{array}{l}1.76 \mathrm{E}-05 \\
1.48 \mathrm{E}-05 \\
1.53 \mathrm{E}-05 \\
2.03 \mathrm{E}-05 \\
1.79 \mathrm{E}-05 \\
2.44 \mathrm{E}-05 \\
2.32 \mathrm{E}-05\end{array}$ & $\begin{array}{l}1.76 \mathrm{E}-05 \mathrm{G} \\
1.48 \mathrm{E}-05 \mathrm{G} \\
1.53 \mathrm{E}-05 \mathrm{E} \\
2.03 \mathrm{E}-05 \mathrm{G} \\
1.79 \mathrm{E}-05 \mathrm{G} \\
2.44 \mathrm{E}-05 \mathrm{G} \\
2.32 \mathrm{E}-05 \mathrm{G}\end{array}$ & $\begin{array}{l}2.65 \\
4.70 \\
0.89 \\
4.70 \\
4.70 \\
2.65 \\
2.65\end{array}$ \\
\hline
\end{tabular}


WHC-SD-FF-TA-040

Rev. 0

Page 13 of 18

\section{GENII Input File \\ Offsite Receptor $7.2 \mathrm{~km} \mathrm{E}$}

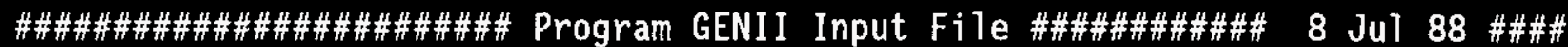
Title: fftf fuel cask drop - 1 assembly - OS $100 \mathrm{~m} \mathrm{~S}$ or $N-150,000$ MWD/MTHM $\backslash$ GENII $\backslash$ fftf2os. in

Created on 08-15-1995 at 10:17

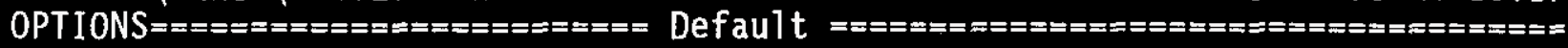

$\begin{array}{llll}F & \text { Near-field scenario? } & \text { (Far-field) } & \text { NEAR-FIELD: narrowly-focused } \\ F & \text { Population dose? } & \text { (Individual) } & \end{array}$

$\mathrm{T}$ Acute release? (Chronic)

Maximum Individual data set used Complete

TRANSPORT OPTIONS $== \pm=== \pm==== \pm$ Section

$T$ Air Transport

ion

F Surface Water Transport

F Biotic Transport (near-field) 3,4

$F$ Waste Form Degradation (near) 3,4

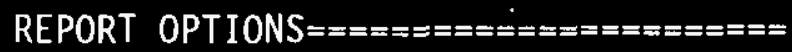

$T$ Report AEDE only

$T$ Report by radionuclide

$T$ Report by exposure pathway

$F$ Debug report on screen

FAR-FIELD: wide-scale release, multiple sites

Complete

EXPOSURE PATHWAY OPTIONS $=====$ Section

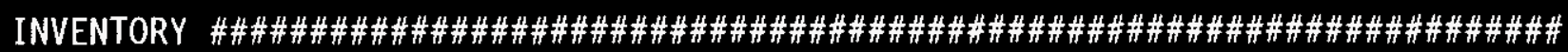

4 Inventory input activity units: (1-pCi $2-\mathrm{uCi} \quad 3-\mathrm{mCi} \quad 4-\mathrm{Ci} \quad 5-\mathrm{Bq}$ )

0 Surface soil source units (1- m2 2- 33 3- $\mathrm{kg}$ )

Equilibrium question goes here

\begin{tabular}{|c|c|c|c|c|c|c|c|c|}
\hline Use when & & $\begin{array}{l}\text { ease Terr } \\
\text { port sel }\end{array}$ & ected & & $\begin{array}{l}\text {-Basic } \\
\text { field sc }\end{array}$ & $\begin{array}{l}\text { Conce } \\
\text { enaric }\end{array}$ & $\begin{array}{l}\text { rations- } \\
\text { optiona } 7\end{array}$ & \\
\hline $\begin{array}{l}\text { Release } \\
\text { Radio- } \\
\text { nuclide }\end{array}$ & $\begin{array}{l}A i r \\
\text { yr }\end{array}$ & $\begin{array}{l}\text { Surface } \\
\text { Water } \\
\text { /yr }\end{array}$ & $\begin{array}{l}\text { Buried } \\
\text { Waste } \\
\text { /m3 }\end{array}$ & $\begin{array}{l}A i r \\
/ m 3\end{array}$ & $\begin{array}{l}\text { Surface } \\
\text { Soi? } \\
\text { /unit }\end{array}$ & $\begin{array}{l}\text { Deep } \\
\text { Soi1 } \\
/ \mathrm{m} 3\end{array}$ & $\begin{array}{l}\text { Ground } \\
\text { Water } \\
/ L\end{array}$ & $\begin{array}{l}\text { Surface } \\
\text { Water } \\
\text { /L }\end{array}$ \\
\hline
\end{tabular}

$\begin{array}{ll}\mathrm{H} 3 & 7.82 \mathrm{E}+1\end{array}$

KR85 $6.74 E+2$

SR90 2.52E-4

$Y 90 \quad 2.53 E-4$

TC99 9.95E-8

RU106 2.45E-4

AGI10M 4.45E-7

CD113M 6.45E-5

SB125 6.05E-5

TE125M $1.48 \mathrm{E}-5$

I $129 \quad 3.32 \mathrm{E}-4$

CS134 1.26E-2

CS137 6.85E-2 
WHC-SD-FF-TA-040

Rev. 0

Page 14 of 18

$\begin{array}{ll}\text { CE144 } & 8.30 E-5 \\ \text { PR144 } & 8.30 E-5 \\ \text { PR144M } & 9.95 E-7 \\ \text { PM147 } & 4.37 E-4 \\ \text { SM151 } & 2.61 E-5 \\ \text { EU154 } & 2.54 E-5 \\ \text { EU155 } & 5.75 E-5 \\ \text { PU238 } & 1.41 E-5 \\ \text { PU239 } & 1.75 E-5 \\ \text { PU240 } & 1.34 E-5 \\ \text { PU241 } & 7.30 E-4 \\ \text { AM241 } & 1.27 E-5 \\ \text { AM242M } & 9.65 E-7 \\ \text { AM242 } & 9.65 E-7 \\ \text { CM242 } & 1.02 E-6 \\ \text { CM244 } & 1.02 E-6\end{array}$

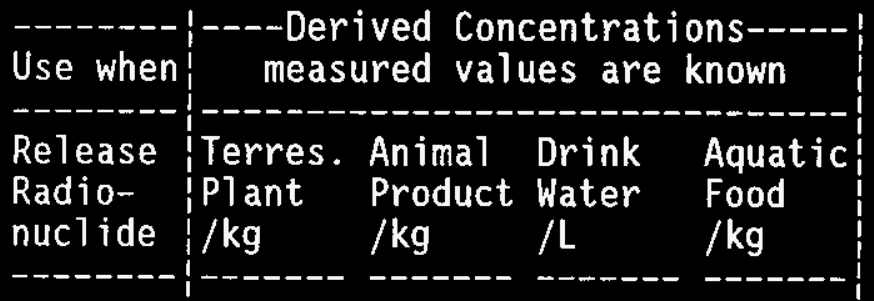

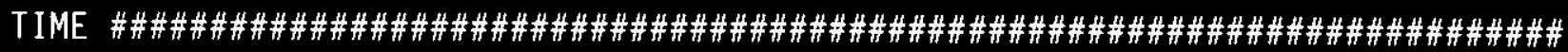

$1 \quad$ Intake ends after (yr)

50 Dose calc. ends after (yr)

0 Release ends after $(y r)$

0 No. of years of air deposition prior to the intake period

0 No. of years of irrigation water deposition prior to the intake period

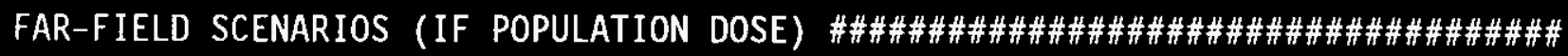

$0 \quad$ Definition option: 1-Use population grid in file POP. IN

0

2-Use total entered on this line

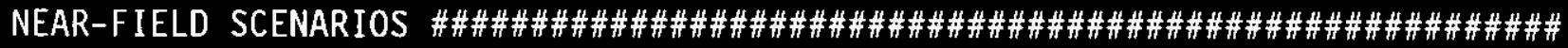

Prior to the beginning of the intake period: (yr)

When was the inventory disposed? (Package degradation starts)

When was LOIC? (Biotic transport starts)

Fraction of roots in upper soil (top $15 \mathrm{~cm}$ )

Fraction of roots in deep soil

Manual redistribution: deep soil/surface soil dilution factor

Source area for external dose modification factor (m2)

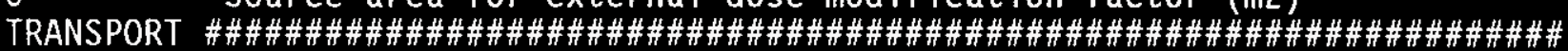

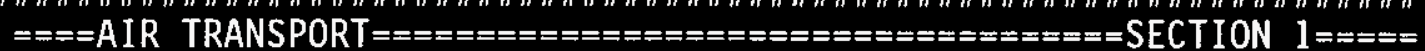

0 -Calculate PM

10 Release type $(0-3)$ 
WHC-SD-FF-TA-040

Rev. 0

Page 15 of 18

3.17E-2

0

0

$\mathrm{F}$

0

0

0

0

0

0

0

0

0

0

0

0

0

$\mathrm{T}$

$\mathrm{T}$

0
Option: 1-Use chi/Q or PM value 2-Select MI dist \& dir 3-Specify MI dist \& dir Chi/Q or PM value

MI sector index (1=S)

MI distance from release point (m) 0 Use jf data, (T/F) else chi/Q gridjo
Stack release (T/F)

Stack height (m)

Stack flow $(\mathrm{m} 3 / \mathrm{sec})$

Stack radius (m)

Effluent temp. (C)

Building $x$-section (m2)

Building height $(\mathrm{m})$

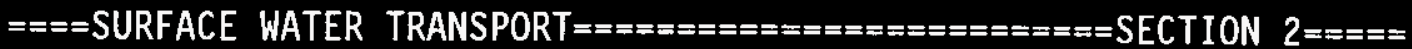
Mixing ratio model: 0-use value, l-river, 2-lake

Mixing ratio, dimensionless

Average river flow rate for: MIXFLG $=0(\mathrm{~m} 3 / \mathrm{s}), \operatorname{MIXFLG}=1,2(\mathrm{~m} / \mathrm{s})$,

Transit time to irrigation withdrawl location (hr)

If mixing ratio model $>0$ :

Rate of effluent discharge to receiving water body $(\mathrm{m} 3 / \mathrm{s})$

Longshore distance from release point to usage location (m)

Offshore distance to the water intake (m)

Average water depth in surface water body $(\mathrm{m})$

Average river width (m), MIXFLG $=1$ only

Depth of effluent discharge point to surface water $(\mathrm{m})$, lake only

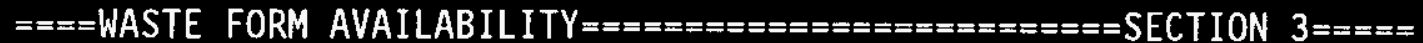

Waste form/package half 1 ife, (yr)

Waste thickness, (m)

Depth of soil overburden, $m$

$===$ BIOTIC TRANSPORT OF BURIED SOURCE $=== \pm== \pm== \pm== \pm=$ SECTION $4== \pm==$ Consider during inventory decay/buildup period (T/F)?

Consider during intake period (T/F)?

Pre-Intake site condition............. 2-Humid non agricultural

3-Agricul tura 1

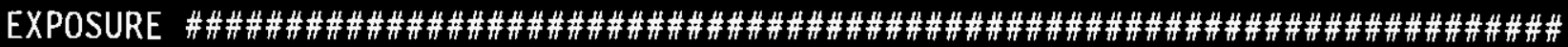

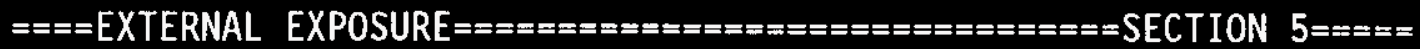
Exposure time:

Plume (hr)

Soil contamination (hr)

Swimming (hr)

Boating (hr)

Residential irrigation:

T Consider: ( $T / F)$

0 Source: 1-ground water

2-surface water

Shoreline activities (hr) 0 Duration (mo/yr)

Shorel ine type: (1-river, 2-lake, 3-ocean, 4-tidal basin)

Transit time for release to reach aquatic recreation (hr)

Average fraction of time submersed in acute cloud (hr/person hr)

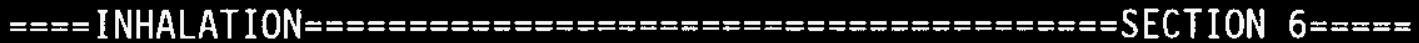

8766.0 Hours of exposure to contamination per year

0

0-No resus- 1-Use Mass Loading 2-Use Anspaugh model

pension Mass loading factor $(\mathrm{g} / \mathrm{m} 3)$ Top soil available $(\mathrm{cm})$ 
WHC-SD-FF-TA-040

Rev. 0

Page 16 of 18

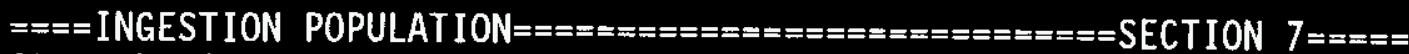
Atmospheric production definition (select option):

0 -Use food-weighted chilQ, (food-sec/m3), enter value on this 1 ine 1-Use population-weighted chi/Q

2-Use uniform production

3-Use chi/Q and production grids (PRODUCTION will be overridden)

Population ingesting aquatic foods, 0 defaults to total (person)

Population ingesting drinking water, 0 defaults to total (person)

Consider dose from food exported out of region (default=F)

Note below: $S^{*}$ or Source: 0 -none, 1-ground water, 2-surface water 3-Derived concentration entered above

$===$ AQUATIC FOODS / DRINKING WATER INGESTION $=======$ SECTION $8====$

Salt water? (default is fresh)

\begin{tabular}{|c|c|c|c|c|c|}
\hline $\begin{array}{ll}\text { USE } & \\
? & \text { FOOD } \\
\text { T/F } & \text { TYPE }\end{array}$ & $\begin{array}{l}\text { TRAN- } \\
\text { SIT } \\
h r\end{array}$ & $\begin{array}{l}\text { PROD- } \\
\text { UCTION } \\
\mathrm{kg} / \mathrm{yr}\end{array}$ & $\begin{array}{l}\text {-CONSUM } \\
\text { HOLDUP } \\
\mathrm{da}\end{array}$ & $\begin{array}{l}\text { TION- } \\
\text { RATE } \\
\mathrm{kg} / \mathrm{yr}\end{array}$ & DRINKING WATER \\
\hline $\begin{array}{l}\text { FISH } \\
\text { MOLLUS } \\
\text { CRUSTA } \\
\text { PLANTS }\end{array}$ & $\begin{array}{l}0.00 \\
0.00 \\
0.00 \\
0.00\end{array}$ & $\begin{array}{l}0.0 E+00 \\
0.0 E+00 \\
0.0 E+00 \\
0.0 E+00\end{array}$ & $\begin{array}{l}0.00 \\
0.00 \\
0.00 \\
0.00\end{array}$ & $\begin{array}{l}0.0 \\
0.0 \\
0.0 \\
0.0\end{array}$ & $\begin{array}{l}\text { Source (see above) } \\
\text { Treatment? T/F } \\
\text { Holdup/trans it (da) } \\
\text { Consumption (L/yr) }\end{array}$ \\
\hline
\end{tabular}

$====$ TERRESTRIAL FOOD INGESTION=

$=$ SECTION $9== \pm==$

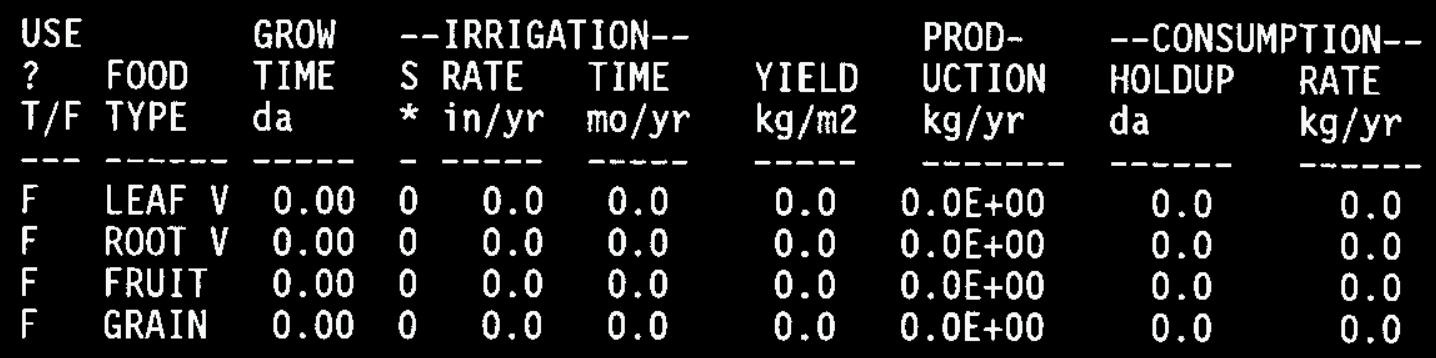

$===$ ANIMAL PRODUCTION CONSUMPTION=

-- HUMAN---- TOTAL
CONSUMPTION PROD-

RATE HOLDUP UCTION

$\mathrm{kg} / \mathrm{yr}$ da $\mathrm{kg} / \mathrm{yr}$

T/F TYPE

-----

BEEF

POULTR

MILK

EGG

BEEF

MILK

\begin{tabular}{|c|c|c|c|c|c|c|c|}
\hline $\begin{array}{l}\text { DRINK } \\
\text { WATER } \\
\text { CONTAM } \\
\text { FRACT. }\end{array}$ & $\begin{array}{l}\text { DIET } \\
\text { FRAC- } \\
\text { TION }\end{array}$ & $\begin{array}{l}\text { GROW } \\
\text { TIME } \\
\mathrm{da}\end{array}$ & $\begin{array}{l}-1 \\
\text { S } \\
\star\end{array}$ & $\begin{array}{l}\text { TORED } \\
\text { RRIGA } \\
\text { RATE } \\
\text { in } / y r\end{array}$ & $\begin{array}{l}\text { FEED-- } \\
\text { TION-- } \\
\text { TIME } \\
\text { mo/yr }\end{array}$ & $\begin{array}{l}\text { YIELD } \\
\mathrm{kg} / \mathrm{m} 3\end{array}$ & $\begin{array}{l}\text { ST0 } \\
\text { AGE } \\
\text { da }\end{array}$ \\
\hline $\begin{array}{l}0.00 \\
0.00 \\
0.00 \\
0.00\end{array}$ & $\begin{array}{l}\overline{0.00} \\
0.00 \\
0.00 \\
0.00\end{array}$ & $\begin{array}{l}0.0 \\
0.0 \\
0.0 \\
0.0\end{array}$ & $\begin{array}{l}0 \\
0 \\
0 \\
0\end{array}$ & $\begin{array}{l}0.0 \\
0.0 \\
0.0 \\
0.0\end{array}$ & $\begin{array}{l}0.00 \\
0.00 \\
0.00 \\
0.00\end{array}$ & $\begin{array}{l}0.00 \\
0.00 \\
0.00 \\
0.00\end{array}$ & $\begin{array}{l}0.0 \\
0.0 \\
0.0 \\
0.0\end{array}$ \\
\hline & $\begin{array}{l}0.00 \\
0.00\end{array}$ & $\begin{array}{l}0.0 \\
0.0\end{array}$ & $\begin{array}{l}0 \\
0\end{array}$ & $\begin{array}{l}0.0 \\
0.0\end{array}$ & $\begin{array}{l}0.00 \\
0.00\end{array}$ & $\begin{array}{l}0.00 \\
0.00\end{array}$ & $\begin{array}{l}0.0 \\
0.0\end{array}$ \\
\hline
\end{tabular}


WHC-SD-FF-TA-040

Rev. 0

Page 17 of 18

\section{CHECKLIST FOR PEER REVIEW}

Document Reviewed: RADIOLOGICAL CONSEQUENCES OF A POSTULATED DROP OF A MAXIMALLY LOADED FFTF FUEL CASK, WHC-SD-FF-TA-040, Rev. 0, D.A. Himes, $8 / 21 / 95$

Scope of Review: entire document

$\frac{\text { Yes No NA }}{\text { [X] [ ] [ ] * }}$

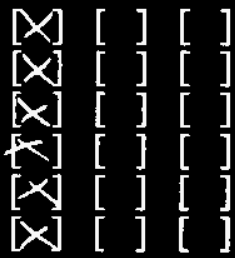

[ ] [ ] [X]

$[\times][$ ] [ ]

W] [ ] [ ]

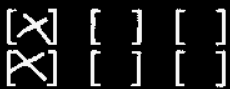

$\mathbb{k}][\mathrm{c}]$

$\underset{\infty}{\infty}\left[\begin{array}{lll}{[} & {[}\end{array}\right]$

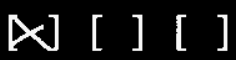

[ ] [ ] $\mathbb{x}]$

[.] $\ll]$ *

め [ ] [ ] Document approved.

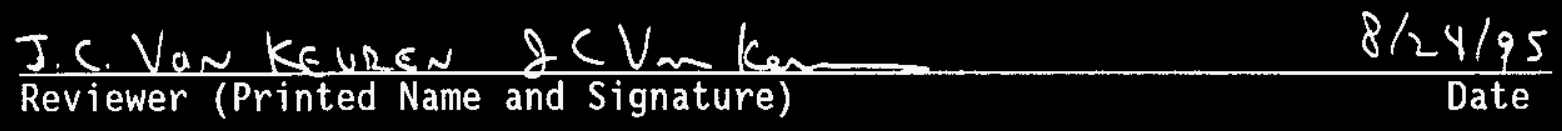

Previous reviews complete and cover analysis, up to scope of this review, with no gaps.

Problem completely defined.

Accident scenarios developed in a clear and logical manner.

Necessary assumptions explicitly stated and supported.

Computer codes and data files documented.

Data used in calculations explicitly stated in document.

Data checked for consistency with original source information as applicable.

Mathematical derivations checked including dimensional

consistency of results.

Models appropriate and used within range of validity or use outside range of established validity justified.

Hand calculations checked for errors. Spreadsheet results should be treated exactly the same as hand calculations.

Software input correct and consistent with document reviewed.

Software output consistent with input and with results reported in document reviewed.

Limits/criteria/guidelines applied to analysis results are appropriate and referenced. Limits/criteria/guidelines checked against references.

Safety margins consistent with good engineering practices.

Conclusions consistent with analytical results and applicable limits.

Results and conclusions address all points required in the problem statement.

Format consistent with appropriate NRC Regulatory Guide or other standards

Review calculations, comments, and/or notes are attached.

* Any calculations, comments, or notes generated as part of this review should be signed, dated and attached to this checklist. Such material should be labeled and recorded in such a manner as to be intelligible to a technically qualified third party. 
WHC-SD-FF-TA-040

Rev. 0

Page 18 of 18

\section{HEDOP REVIEN CHECKLIST \\ for \\ Radiological and Nonradiological Release Calculations}

Document reviewed (include title or description of calculation, document number, author, and date, as applicable):

RADIOLOGICAL CONSEQUENCES OF A POSTULATED DROP OF A MAXIMALLY LOADED

FFTF FUEL CASK, WHC-SD-FF-TA-040, Rev. 0, D.A. Himes, 8/21/95

Submitted by: D.A. Himes

Date Submitted: $8 / 28 / 95$

Scope of Review: entire document

$\underline{\text { YES }} \quad \mathrm{NO} 0^{*} \quad \mathrm{~N} / \mathrm{A}$

[x] [ ] [ ] 1. A detailed technical review and approval of the environmental transport and dose calculation portion of the analysis has been performed and documented.

[ ] [ ] [ [X] 2. Detailed technical review(s) and approval(s) of scenario and release determinations have been performed and documented.

$[X]$ [ ] [ ] 3. HEDOP-approved code(s) were used.

$[x]$ [ ] [ ] 4. Receptor locations were selected according to HEDOP recommendations.

[X] [ ] [ ] 5. All applicable environmental pathways and code options were included and are appropriate for the calculations.

$[X]$ [ ] [ ] 6. Hanford site data were used.

$\left[\begin{array}{lll}{[} & {[}\end{array}\right][X]$ 7. Model adjustments external to the computer program were justified and performed correctly.

[ $\mathrm{x}]$ [ ] [ ] 8. The analys is is consistent with HEDOP recommendations.

[]$\quad[x]$ 9. Supporting notes, calculations, comments, comment resolutions, or other information is attached. (Use the "Page 1 of $X$ " page numbering format and sign and date each added page.)

$[x] \quad[\quad]$

10. Approval is granted on behalf of the Hanford Environmental Dose Overview Panel.

* A11 "NO" responses must be explained and use of nonstandard methods justified.

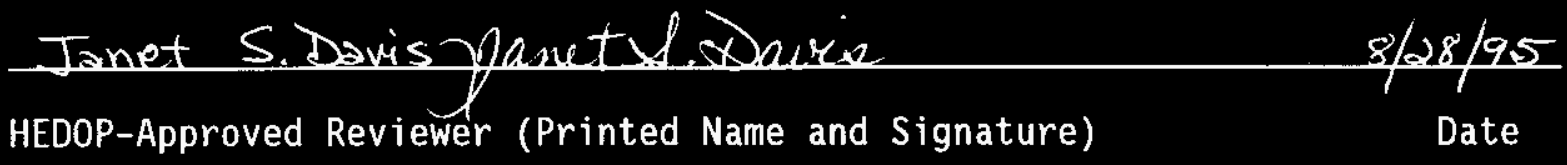

COMMENTS (add additional signed and dated pages if necessary):

2. Release determintions are based on inventories provided in previous onalyses. 3. GXQ (nor HEDOP-approved) was used to colculate X/Qs becouse Gendi does not coloulate MQs in accordance with MRC Regulatory Guide 1.145. GENII (HEDOP-approved) was wed for dose calculations. 